\title{
NEARLY DIRECTED SUBSPACES OF PARTIALLY ORDERED LINEAR SPACES
}

\author{
by G. J. O. JAMESON \\ (Received 1st December 1967)
}

\section{Introduction}

Let $X$ be a partially ordered linear space, i.e. a real linear space with a reflexive, transitive relation $\leqq$ such that

$$
\begin{gathered}
x \leqq y \text { implies } x+z \leqq y+z \text { for all } z \in X, \\
x \geqq 0, \lambda \geqq 0 \text { implies } \lambda x \geqq 0 .
\end{gathered}
$$

The positive wedge, i.e. $\{x: x \geqq 0\}$, will be denoted consistently by $P$. Clearly, $x \leqq y$ is equivalent to $y-x \in P$.

A subset $A$ of $X$ is order-convex (in $X$ ) if $a, b \in A$ and $a \leqq x \leqq b$ imply $x \in A$. If $A$ is a linear subspace, it is order-convex if and only if $a, b \in P$ and $a+b \in A$ implies that $a, b \in A$. If $Y$ is a subspace of $X$ and $A \subseteq Y$, then $A$ is order-convex in $Y$ if $a, b \in A, y \in Y$ and $a \leqq y \leqq b$ implies that $y \in A$. The following facts about order-convexity in subspaces are elementary:

(i) if $Y$ is a subspace of $X$, and $A$ is order-convex in $X$, then $A \cap Y$ is orderconvex in $Y$;

(ii) if $Y$ is order-convex in $X$, and $A \subseteq Y$ is order-convex in $Y$, then $A$ is order-convex in $X$.

If $Y$ is not order-convex in $X$, then some subsets of $Y$ (for instance, $Y$ itself) are order-convex in $Y$ but not in $X$.

A linear subspace $E$ of $X$ is directed if $E \cap P-E \cap P=E$. Equivalently, given $x, y \in E$, there exists $z \in E$ such that $z \geqq x$ and $z \geqq y$.

A positive element $e$ is an order-unit if, given $x \in X$, there exists $\lambda>0$ such that $x \leqq \lambda e$. Equivalently, $e$ is an internal point of $P$.

We shall denote by $X^{\prime}$ the space of all linear functionals on $X$. For $A \subseteq X$, write

$$
\begin{gathered}
A^{+}=\left\{f \in X^{\prime}: f(a) \geqq 0 \text { for all } a \in A\right\}, \\
A^{\circ}=\left\{f \in X^{\prime}:|f(a)| \leqq 1 \text { for all } a \in A\right\},
\end{gathered}
$$

and for $B \subseteq X^{\prime}$, write

$$
B_{0}=\{x \in X:|f(x)| \leqq 1 \text { for all } f \in B\} .
$$

The natural ordering of $X^{\prime}$ (which will be denoted by the same notation $\leqq$ ) is that associated with the wedge $P^{+}$. Clearly, if $E$ is a linear subspace of $X$, then $E^{+}, E^{\circ}$ are both the set of linear functionals that vanish on $E$. 
If $\tau$ is a linear topology for $X$, we shall denote by $\mathcal{N}(\tau)$ the set of all $\tau$ neighbourhoods of 0 , and by $(X, \tau)^{*}$, or just $X^{*}$, the space of all $\tau$-continuous linear functionals. If $Y$ is a linear subspace of $X^{\prime}$, we shall denote by $\sigma(Y)$ the weak topology induced by $Y$ on $X$ and its subspaces. In general, $X^{*}$ need not be order-convex in $X^{\prime}$ (see Example (i)); however, this is the case if $P$ gives an open decomposition of $X$, i.e. if $P \cap U-P \cap U \in \mathcal{N}(\tau)$ for each $U \in \mathcal{N}(\tau)$.

It is elementary that if a linear subspace $E$ of $X$ is directed, then $E^{\circ}$ is orderconvex in $X^{\prime}$. The converse, however, is not true (see Example (ii)). In the present paper, a topological notion, " nearly directed", is introduced which enables us to characterise not only those subspaces $E$ of $X$ for which $E^{\circ}$ is order-convex in $X^{\prime}$, but also those for which, in the presence of a locally convex on $X, E^{\circ} \cap X^{*}$ is order-convex in $X^{*}$. A similar result was proved by Ellis (3), subject to certain superfluous conditions. Our methods are quite different from those of Ellis (they were, in fact, developed in ignorance of Ellis's paper). In (3), the term " perfect " is used for a property which, in locally convex spaces, is equivalent to our " nearly directed". "Perfect" order-convex subspaces were first defined by Bonsall (1) in the context of partially ordered linear spaces with an order-unit. A different, non-topological generalisation of Bonsall's definition was used by Kist (6). We shall use the word perfect to mean " perfect in the sense of Kist "; the relation between this property and " nearly directed" is considered in $\S 6$.

If $x \in P \sim\{0\}$, then the one-dimensional subspace spanned by $x$ is orderconvex if and only if $0 \leqq y \leqq x$ implies that $y$ is a scalar multiple of $x$. We shall say that $x$ is an extremal element of $P$ in this case (thus making a distinction between extreme and extremal). Our result clearly enables us to characterise extremal elements of $P^{+} \cap X^{*}$ by their kernels. Another characterisation, showing in a sense that extremal elements of $P^{+}$are " nearly" lattice homomorphisms, follows with ease (Theorem 2); Hayes (4) has proved a version of this result applicable to commutative groups.

I am grateful to Professor Bonsall for drawing my attention to (4) and for providing Example (ii) (although with a different purpose from that for which it is used here).

\section{Definition and elementary properties}

Definition. Let $(X, \tau)$ be a topological linear space with a partial ordering $\leqq$. A linear subspace $E$ is nearly directed (in $X$ ) with respect to $\tau$ if, given $x \in E$ and $U \in \mathcal{N}(\tau)$, there exist $y \in E$ and $u_{1}, u_{2} \in U$ such that

$$
-\left(y+u_{2}\right) \leqq x \leqq y+u_{1} .
$$

Clearly, if $E$ is nearly directed with respect to $\tau$, then it is nearly directed with respect to any smaller topology for $X$.

Lemma 1. $E$ is nearly directed with respect to $\tau$ if and only if, given $x \in E$ and $U \in \mathcal{N}(\tau)$, there exist $y \in E$ and $u_{1}, u_{2} \in U$ such that $y \geqq x+u_{1}$ and $y \geqq u_{2}$. 
Proof. (i) Suppose that $E$ is nearly directed and that $U \in \mathcal{N}(\tau)$ is given. Take circled $V \in \mathscr{N}(\tau)$ such that $V+V \subseteq U$. There exist $y \in E$ and $v_{1}, v_{2} \in V$ such that

$-\left(y+v_{2}\right) \leqq x \leqq y+v_{1}$,
or $y \geqq x-v_{1}$ and $y \geqq-x-v_{2}$. Then $y-x \geqq-v_{1} \in U$ and

as required.

$$
y \geqq-\frac{1}{2}\left(v_{1}+v_{2}\right) \in U \text {, }
$$

(ii) Suppose that the condition holds and $U \in \mathscr{N}(\tau)$ is given. Take circled $V \in \mathcal{N}(\tau)$ such that $V+V \subseteq U$. There exist $y \in E$ and $v_{1}, v_{2} \in V$ such that $y \geqq x+v_{1}$ and $y \geqq v_{2}$. Also, there exist $z \in E$ and $w_{1}, w_{2} \in V$ such that $z \geqq-x+w_{1}$ and $z \geqq w_{2}$. Then

showing that $E$ is nearly directed.

$$
-y-z+v_{2}+w_{1} \leqq x \leqq y+z-v_{1}-w_{2},
$$

Corollary 1. If $E$ is nearly directed with respect to $\tau$, then $E$ is contained in the $\tau$-closure of $P-P$.

Proof. Given $x \in E$ and $U \in \mathscr{N}(\tau)$, take $V \in \mathcal{N}(\tau)$ such that $V-V \subseteq U$. There exist $y \in E, v_{1}, v_{2} \in V$ and $p, q \in P$ such that $x+v_{1}=y-p$ and $v_{2}=y-q$. Then $x+\left(v_{1}-v_{2}\right)=q-p$, i.e. $x+U$ meets $P-P$.

Corollary 2. $X$ is nearly directed if and only if $P-P$ is dense in $X$.

Proof. Suppose that $P-P$ is dense in $X$. Take $x \in X$ and $U \in \mathscr{N}(\tau)$. There exist $p, q \in P$ and $u \in U$ such that $x+u=p-q$. Then $p \geqq 0$ and $p \geqq x+u$, so $X$ is nearly directed.

Example (ii) below shows that $E$ can be nearly directed (in $X$ ) without containing any non-zero positive elements.

Lemma 2. Suppose that $P$ gives an open decomposition of $X$ with respect to $\tau$. Then a linear subspace $E$ is nearly directed with respect to $\tau$ if and only if, given $x \in E$ and $U \in \mathscr{N}(\tau)$, there exist $y \in E$ and $u \in U$ such that $y+u \geqq x$ and $y+u \geqq 0$.

Proof. The condition is clearly sufficient. Suppose that $E$ is nearly directed, and take $x \in X$ and $U \in \mathcal{N}(\tau)$. There exists $U_{1} \in \mathcal{N}(\tau)$ such that $U_{1}+U_{1} \subseteq U$. Let $V=P \cap U_{1}-P \cap U_{1}$. Then there exist $y \in E$ and $v_{1}, v_{2} \in V$ such that $y \geqq x+v_{1}$ and $y \geqq v_{2}$. Now $v_{i}=u_{i}-u_{i}^{\prime}$ for some $u_{i}, u_{i}^{\prime} \in P \cap U_{1}(i=1,2)$. Let $u=u_{1}^{\prime}+u_{2}^{\prime}$. Then $u \in U$ and $y+u \geqq x, y+u \geqq 0$.

We shall say that a set is radial if it is radial at 0 (absorbing). The largest locally convex topology for $X$ is that in which all convex, radial sets are neighbourhoods of 0 . With respect to this topology, all linear functionals are continuous and all linear subspaces are closed (see (7), p. 69). Also, it is clear that if $P-P=X$, then $P$ gives an open decomposition with respect to this topology. Hence we have:

Corollary. Suppose that $P-P=X$. Then $E$ is nearly directed with respect to the largest locally convex topology for $X$ if and only if, given $x \in E$ and a convex, radial set $U$, there exist $y \in E$ and $u \in U$ such that $y+u \geqq x$ and $y+u \geqq 0$.

$$
\text { E.M.S. }-\mathrm{K}
$$




\section{The main theorem}

Theorem 1. Let $X$ be a partially ordered linear space with a locally convex topology $\tau$, and let $E$ be a linear subspace of $X$. Then the following statements are equivalent:

(i) $E$ is nearly directed with respect to $\tau$;

(ii) $E^{\circ} \cap X^{*}$ is order-convex in $X^{*}$.

Proof. (i) $\Rightarrow$ (ii). Suppose that $f \in E^{\circ} \cap X^{*}, g \in X^{*}$ and $0 \leqq g \leqq f$. Take $x \in E$ and $\varepsilon>0$. There exists $U \in \mathscr{N}(\tau)$ such that $|f(u)|$ and $|g(u)| \leqq \varepsilon$ for $u \in U$. There exist $y \in E$ and $u_{1}, u_{2} \in U$ such that $y \geqq x+u_{1}$ and $y \geqq u_{2}$. Then

$$
g(x) \leqq g\left(y-u_{1}\right) \leqq g\left(y-u_{2}\right)+2 \varepsilon \leqq f\left(y-u_{2}\right)+2 \varepsilon \leqq 3 \varepsilon .
$$

Hence $g(x) \leqq 0$ for $x \in E$, so $g \in E^{\circ}$.

(ii) $\Rightarrow$ (i). Suppose that $E$ is not nearly directed, and consider the space $X \times X$. There exist $x_{0} \in E$ and $U \in \mathscr{N}(\tau)$ such that, given $u_{1}, u_{2} \in U, y \in E$ and $p, q \in P$,

Write

$$
\left(x_{0},-x_{0}\right)+\left(u_{1}, u_{2}\right) \neq(y-p, y-q) \text {. }
$$

$$
H=\{(y-p, y-q): y \in E \text { and } p, q \in P\} .
$$

Then $H$ is a wedge and $\left(x_{0},-x_{0}\right)$ is not in the closure of $H$. Hence there is a continuous linear functional $\phi$ on $X \times X$ such that $\phi\left(x_{0},-x_{0}\right)>\sup \phi(H)$. Since $H$ is a wedge, this implies that $\phi \leqq 0$ on $H$. Let $g(x)=\phi(x, 0)$, $h(x)=\phi(0, x)(x \in X)$. Then $g, h \in X^{*}$ and $\phi(x, y)=g(x)+h(y)$. For $p \in P$, $g(-p)=\phi(-p, 0) \leqq 0$, so $g \in P^{+}$. Similarly, $h \in P^{+}$. For $y \in E$,

$$
\phi(y, y)=g(y)+h(y) \leqq 0, \text { so } g+h \in E^{\circ} .
$$

But $g\left(x_{0}\right) \neq h\left(x_{0}\right)$, so $g$ and $h$ are not in $E^{\circ}$. It follows that $E^{\circ} \cap X^{*}$ is not order-convex in $X^{*}$.

Corollary 1. $E^{\circ}$ is order-convex in $X^{\prime}$ if and only if $E$ is nearly directed with respect to the largest locally convex topology for $X$.

Corollary 2. $E$ is nearly directed with respect to a locally convex topology $\tau$ if and only if it is nearly directed with respect to the associated weak topology.

Proof. If $E$ is nearly directed with respect to the weak topology, then $E^{\circ} \cap X^{*}$ is order-convex in $X^{*}$, so $E$ is nearly directed with respect to $\tau$.

Corollary 3. An element $f$ of $P^{+} \cap X^{*}$ is extremal in $P^{+} \cap X^{*}$ if and only if its kernel is nearly directed.

Corollary 4. Suppose that $E$ and $P$ are $\tau$-closed. Then $E$ is order-convex if and only if $E^{\circ} \cap X^{*}$ is nearly directed with respect to $\sigma(X)$.

Proof. Consider the space $X^{*}$ with topology $\sigma(X)$ and positive wedge $P^{+}$. The dual space can be identified with $X$, and the set of positive continuous linear functionals is $P$, since $P$ is closed. Also, $\left(E^{\circ} \cap X^{*}\right)_{0}=E$. The result follows. 
Corollary 5. Suppose that $P$ is closed with respect to the largest locally convex topology for $X$, and let $E$ be any linear subspace of $X$. Then $E$ is orderconvex if and only if $E^{\circ}$ is nearly directed with respect to $\sigma(X)$.

Example (iv) below shows that corollary 4 can fail if $P$ is not closed.

A local base for the order-bound topology for $X$ is the family of convex, symmetric sets that absorb all order-intervals. We denote this topology by $\tau_{b}$. Some properties of this topology (called by Schaefer the "order topology") are given in (7), ch. 5. It is easily seen that if $P-P=X$, then $P$ gives an open decomposition of $X$ with respect to $\tau_{b}$. The $\tau_{b}$-continuous linear functionals are those that are bounded on order-intervals. Denote the set of these by $X^{b}$. Clearly, all positive linear functionals are in $X^{b}$, so if $E^{\circ} \cap X^{b}$ is order-convex in $X^{b}$, then $E^{\circ}$ is order-convex in $X^{\prime}$. Hence we have:

Corollary 6. If $E$ is nearly directed with respect to $\tau_{b}$, then $E$ is nearly directed with respect to all locally convex topologies on $X$.

The order-bound topology coincides with the usual topology in quite a wide range of spaces (when given their "natural" orders), so Corollary 6 is of some interest.

\section{Extremal positive functionals}

Using Corollary 3 to Theorem 1, we can give the following purely algebraic characterisation of extremal elements of $P^{+}$(cf. Hayes (4)). No analogous result seems to be available for extremal elements of $P^{+} \cap X^{*}$, but these are, of course, extremal in $P^{+}$whenever $P$ gives an open decomposition of $X$.

Theorem 2. Let $X$ be a partially ordered linear space, and suppose that $P-P=X$, where $P$ denotes the positive wedge. Suppose that $f \in P^{+}$, and let $K$ be the kernel of $f$. Then the following statements are equivalent :

(i) $f$ is an extremal element of $P^{+}$;

(ii) given $x \in K$ and $\varepsilon>0$, there exists $a \in X$ such that $a \geqq x, a \geqq 0$ and $f(a) \leqq \varepsilon$

(iii) for any $x, y \in X$, $\inf \{f(z): z \geqq x$ and $z \geqq y\}=\max (f(x), f(y))$.

Proof. (i) $\Rightarrow$ (ii). Take $x \in K$ and $\varepsilon>0$. Let $U=\{u \in X:|f(u)| \leqq \varepsilon\}$. This is a convex, radial set. By Corollary 3 to Theorem 1 and the Corollary to Lemma 2, there exist $y \in K$ and $u \in U$ such that $y+u \geqq x$ and $y+u \geqq 0$. Now $f(y+u) \leqq \varepsilon$, so (ii) holds.

(ii) $\Rightarrow$ (iii). Take $x, y \in X$ and $\varepsilon>0$. Suppose that $f(x-y)=\lambda \geqq 0$. Take $z \in P$ such that $f(z)=1$. Then $x-y-\lambda z \in K$, so, by (ii), there exists $a \in P$ such that $a \geqq x-y-\lambda z$ and $f(a) \leqq \varepsilon$. Let $b=a+y+\lambda z$. Then $b \geqq x$, $b \geqq y$ and $f(b) \leqq f(x)+\varepsilon$. 
(iii) $\Rightarrow$ (i). Suppose that $0 \leqq g \leqq f$ and $f(x)=0$. Take $\varepsilon>0$. There exist $u, v \in P$ such that $x=u-v$. Then $f(u)=f(v)$. By (iii), there exists $a \in X$ such that $a \geqq u, a \geqq v$ and $f(a) \leqq f(u)+\varepsilon$. Then

$$
0 \leqq g(a-u) \leqq f(a-u) \leqq \varepsilon,
$$

and, similarly, $0 \leqq g(a-v) \leqq \varepsilon$. But $x=(a-v)-(a-u)$, so $|g(x)| \leqq \varepsilon$. Hence $g(x)=0$. It follows that $g$ is a scalar multiple of $f$.

(Alternatively, it is quite a simple matter to show that condition (iii) implies that $K$ is nearly directed with respect to the largest locally convex topology).

In the case when $X$ has an order-unit, Bonsall showed that each perfect order-convex subspace is contained in a maximal one, which is the kernel of an extremal element of $P^{+}$. This theory is of particular interest in that it leads to a purely algebraic proof of a purely algebraic form of the Krein-Milman theorem. However, some assumptions are needed to ensure the existence of extremal elements of a cone; for instance, it is well-known that there are no extremal positive functionals on $L_{1}[0,1]$. Two positive results not depending on orderunits are:

(i) a locally compact cone is the closed, convex cover of its extremal elements (see (2));

(ii) if $X$ is a topological $M$-space (see (5)), then the extremal elements of $P^{+}$ separate points of $X$.

\section{Lattices}

Let $X$ be a linear lattice. By a lattice homomorphism we shall mean a linear functional $f$ on $X$ such that

$$
f(x \vee y)=f(x) \vee f(y)
$$

for $x, y \in X$ (the second occurrence of $\vee$ here refers, of course, to the usual lattice ordering of $R$ ). Writing, as usual, $x^{+}=x \vee 0$ and $x^{-}=(-x) \vee 0$, we have $x=x^{+}-x^{-}$. A linear subspace $E$ is. a sublattice if and only if $x \in E$ implies $x^{+} \in E$. Thus a linear sublattice is directed, and it is elementary that a directed, order-convex subspace is a sublattice. Example (ii) shows that a nearly directed, order-convex subspace need not be a sublattice.

It is clear that the kernel of a lattice homomorphism is a sublattice. This fact, together with Theorems 1 and 2 , shows that the following four conditions for $f \in P^{+}$are equivalent:

(i) $f$ is an extremal element of $P^{+}$;

(ii) $f$ is a lattice homomorphism;

(iii) the kernel of $f$ is a sublattice;

(iv) the kernel of $f$ is nearly directed with respect to the largest locally convex topology for $X$.

The equivalence of the first three of these conditions is well-known. The equivalence of (iii) and (iv) can be stated in the following way: in the class of 
order-convex maximal subspaces, all nearly directed subspaces are sublattices. The next theorem shows that this is true in a wider class of subspaces.

Theorem 3. Let $X$ be a linear lattice with a topology. Suppose that the positive wedge $P$ gives an open decomposition of $X$. If $F \subseteq P^{+} \cap X^{*}$, and $F_{0}$ is nearly directed, then $F_{0}$ is a sublattice.

Proof. Take $x \in F_{0}, f \in F$ and $\varepsilon>0$. There exists $U \in \mathcal{N}(\tau)$ such that $|f(u)| \leqq \varepsilon$ for $u \in U$. By Lemma 2, there exist $y \in F_{0}$ and $u \in U$ such that $y+u \geqq x$ and $y+u \geqq 0$. Then $y+u \geqq x^{+}$, so $f\left(x^{+}\right) \leqq \varepsilon$. It follows that $f\left(x^{+}\right)=0$, so that $x^{+} \in F_{0}$.

Corollary. Let $X$ be a linear lattice, and denote the positive wedge by $P$. If $F \subseteq P^{+}$and $F_{0}$ is nearly directed with respect to the largest locally convex topology for $X$, then $F_{0}$ is a sublattice.

If $X$ is a linear lattice, then a sufficient condition for $P$ to give an open decomposition of $X$ is that $x \rightarrow x^{+}$is continuous at 0 . This condition is also necessary if $X$ is locally order-convex (in which case, in fact, $x \rightarrow x^{+}$is uniformly continuous on $X$; see (5)).

\section{Comparison with perfect subspaces}

Let $X$ be a partially ordered linear space with an order-unit $e$. A linear subspace $E$ of $X$ is said to be perfect (Bonsall, (1)) if, given $x \in E$ and $\varepsilon>0$, there exists $y \in E$ such that

$$
-(y+\varepsilon e) \leqq x \leqq y+\varepsilon e .
$$

It is easily checked that the definition is independent of the choice of orderunit.

There is a simple connection between our definition and this one. If $e$ is an order-unit, then a corresponding "order-unit seminorm" is defined as follows :

$$
\|x\|=\inf \{\lambda>0:-\lambda e \leqq x \leqq \lambda e\} .
$$

Equivalent seminorms are obtained from different order-units, and the topology induced by each of them is the order-bound topology. The seminorm is a norm if and only if the ordering is almost Archimedean. The next theorem is stated without proof in (3), but we repeat it (with proof) for completeness.

Theorem 4. Let $X$ be a partially ordered linear space with an order-unit, and let $E$ be a linear subspace of $X$. Then the following statements are equivalent:

(i) $E$ is nearly directed with respect to the order-bound topology;

(ii) $E$ is perfect.

Proof. It is obvious that (ii) implies (i). To show that (i) implies (ii), let $e$ be an order-unit, and let \|\| be the associated seminorm. Suppose that $E$ is nearly directed with respect to the order-bound topology. Then, given $x \in E$ and $\varepsilon>0$, there exist $y \in E$ and $u_{1}, u_{2}$ such that $\left\|u_{i}\right\|<\varepsilon(i=1,2)$ and

$$
-\left(y+u_{2}\right) \leqq x \leqq y+u_{1} \text {. }
$$


Then $u_{i} \leqq \varepsilon e(i=1,2)$, so

and $E$ is perfect.

$$
-(y+\varepsilon e) \leqq x \leqq y+\varepsilon e,
$$

Thus if $X$ has an order-unit and $E$ is nearly directed with respect to the orderbound topology, then $E$ is nearly directed with respect to every linear topology on $X$ (cf. Corollary 6 to Theorem 1 ).

For spaces without an order-unit, Kist (6) generalised Bonsall's definition as follows: $E$ is perfect if there exists $a \in P$ such that, given $x \in E$ and $\varepsilon>0$, there exists $y \in E$ such that

$$
-(y+\varepsilon a) \leqq x \leqq y+\varepsilon a .
$$

This is equivalent to Bonsall's definition in the case when $X$ has an order-unit, and it obviously implies that $E$ is nearly directed with respect to any linear topology. Kist showed that $f$ is an extremal element of $P^{+}$if and only if its kernel is perfect. Thus if $\tau$ is a topology with respect to which $P$ gives an open decomposition, and $E$ is an order-convex maximal subspace that is closed and nearly directed with respect to $\tau$, then $E$ is perfect. The next result shows that this remains true in the absence of order-convexity.

Theorem 5. Let $X$ be a partially ordered linear space with positive wedge $P$ and topology $\tau$, and suppose that $P$ gives a $\tau$-open decomposition of $X$. Suppose that $E$ is a maximal subspace of $X$ that is closed and nearly directed with respect to $\tau$. Then $E$ is perfect.

Proof. $E$ is the kernel of some $f \in X^{*}$. Take $a \in X$ such that $f(a)=1$. Given $x \in E$ and $\varepsilon>0$, there exist $y \in E$ and $u \in X$ such that $|f(u)| \leqq \varepsilon$ and

$$
-(y+u) \leqq x \leqq y+u .
$$

Write $f(u)=\lambda$ and $u-\lambda a=z$. Then $z \in E$, Since $P-P=X$, there exists $b \in P$ such that $-b \leqq a \leqq b$. Now we have

$$
-(y+z+\varepsilon b) \leqq x \leqq y+z+\varepsilon b .
$$

Corollary. If $P-P=X$, then a maximal subspace of $X$ is perfect if and only if it is nearly directed with respect to the largest locally convex topology.

We notice that $X$ itself is perfect if and only if $P-P=X$, so Corollary 2 to Lemma 1 shows that $X$ can easily be nearly directed with respect to a wellbehaved topology without being perfect. An example of a proper subspace with this property is given below (Example (iii)).

\section{Examples}

(i) $X^{*}$ not order-convex in $X^{\prime}$. Let $X$ be the space of finite real sequences with the $l_{1}$-norm:

$$
\left\|\left(\xi_{1}, \ldots, \xi_{n}\right)\right\|=\Sigma\left|\xi_{i}\right| \text {. }
$$

Then $X^{\prime}$ is the space of all real sequences, while $X^{*}$ is the space of all bounded ones. 
Let $P$ be the set of decreasing, non-negative sequences in $X$. Then $P-P=X$, but $P$ does not give an open decomposition of $X$ with respect to the norm. $P^{+}$ is easily seen to be the set of all sequences having each of their partial sums non-negative. This gives a partial ordering of $X^{\prime}$ with respect to which $X^{*}$ is not order-convex, since

$$
\begin{aligned}
0 & \leqq(1,-1,2,-2,3,-3, \ldots) \\
& \leqq(1,0,1,0,1,0, \ldots) .
\end{aligned}
$$

(ii) A perfect, order-convex subspace that is not directed. Let $X$ be the space of all bounded real sequences with the usual norm and order. Let $e$ be the sequence having 1 in each place; then $e$ is an order-unit in $X$. Let $e_{n}$ be the sequence having 1 in place $n$ and 0 elsewhere, and let $a$ be the sequence having 1 in odd places and 0 in even places. Let $x_{n}=a-n^{-1} e_{2 n}$, and let $E$ be the subspace spanned by the $x_{n}$. Then $E \cap P=\{0\}$, so $E$ is order-convex and not directed. Now a general element of $E$ is

$$
x=\sum_{i \in I} \lambda_{i} x_{i}-\sum_{j \in J} \mu_{j} x_{j},
$$

where each $\lambda_{i}, \mu_{j}>0$ and $I, J$ are disjoint, finite sets of integers. Let

$$
\lambda=\sum_{i \in I} \lambda_{i}, \quad \mu=\sum_{j \in J} \mu_{j}
$$

Given $\varepsilon>0$, take $r>(\lambda \vee \mu) \varepsilon^{-1}$, and let

Then

$$
y=(\lambda \vee \mu) x_{r}-\sum_{j \in J} \mu_{j} x_{j}
$$

$$
-(y+\varepsilon e) \leqq x \leqq y+\varepsilon e .
$$

(This subspace has the interesting property that it is order-convex, while its closure is not, a fact pointed out by Professor Bonsall.)

(iii) A subspace that is nearly directed with respect to one topology but not another. Let $X$ be the space of finite real sequences, this time with the usual order. With $e_{n}$ as in example (ii), let $x_{n}=e_{1}-e_{n}(n \geqq 2)$, and let $E$ be the subspace spanned by the $x_{n}$.

Let $p$ be the supremum norm on $X$. Take $\varepsilon>0$, and suppose that a general element $x$ of $E$ is expressed as in (ii). Take a finite set $K$ of integers disjoint from $J$ and corresponding numbers $v_{k}$ such that $0<v_{k}<\varepsilon(k \in K)$, and

Let

$$
\sum_{k \in K} v_{k}=\lambda \vee \mu \text {. }
$$

Then

$$
y=\sum_{k \in K} v_{k} x_{k}-\sum_{j \in J} \mu_{j} x_{j}
$$

$$
y=(\lambda-\mu)^{+} e_{1}-\sum_{k \in K} v_{k} e_{k}+\sum_{j \in J} \mu_{j} e_{j}
$$

Let $u=\varepsilon \sum_{k \in K} e_{k}$. Then $p(u) \leqq \varepsilon, y+u \geqq 0$ and $y+u \geqq x$. Thus $E$ is nearly directed with respect to $p$. 
Let \|\| be the $l_{1}$-norm on $X$. Suppose that $y \in E$ and $u \in X$ are such that $y+u \geqq 0$ and $y+u \geqq-x_{2}$. We show that $\|u\| \geqq \frac{1}{3}$, from which it follows that $E$ is not nearly directed with respect to \|\| . Let

where $\alpha_{i}, \beta_{j}>0$, and write

$$
y=\sum_{i \in I} \alpha_{i} x_{i}-\sum_{j \in J} \beta_{j} x_{j}
$$

$$
\alpha=\sum_{i \in I} \alpha_{i}, \quad \beta=\sum_{j \in J} \beta_{j}
$$

Now $\|u\| \geqq \alpha$, since $y+u \geqq 0$. Suppose that $\|u\|<\frac{1}{3}$. Then $\alpha<\frac{1}{3}$. The first term of $y$ is $\alpha-\beta$, so $\beta<\frac{2}{3}$. But the second term of $y+u$ is not less than 1 , and this clearly leads to a contradiction.

In particular, of course, this subspace is not perfect.

(iv) $E$ order-convex while $E^{\circ}$ is not nearly directed. Let $X$ be the space of finite real sequences, and let $P$ be the set of finite sequences whose last non-zero term is positive. Then $P^{+}=\{0\}$, so no non-zero subspace of $X^{\prime}$ is nearly directed with respect to $\sigma(X)$. However, $X$ has proper order-convex subspaces, for instance the one-dimensional subspace spanned by $e_{1}$.

\section{REFERENCES}

(1) F. F. Bonsall, Extreme maximal ideals of a partially ordered vector space, Proc. Amer. Math. Soc. 7 (1956), 831-837.

(2) D. A. EDWARDs, On the homeomorphic affine embedding of a locally compact cone into a Banach dual space endowed with the vague topology, Proc. London Math. Soc. (3) 14 (1964), 399-414.

(3) A. J. EluIs, Perfect order ideals, J. London Math Soc. 40 (1965), 288-294.

(4) A. HAYEs, Indecomposable positive additive functionals, J. London Math Soc. 41 (1966), 318-322.

(5) G. J. O. JAMESON, Topological M-spaces, Math. Zeitschrift 103 (1968),139-150.

(6) J. KIST, Indecomposable maximal ideals of a partially ordered vector space, J. London Math. Soc. 36 (1961), 436-438.

(7) H. H. SCHAEFER, Topological Vector Spaces (Macmillan, New York 1966).

UNIVERSITY OF EDINBURGH AND

UNIVERSITY OF WARWICK 